\title{
Análisis de mapas causales de impactos del turismo residencial
}

\author{
ANTONIO Aledo \\ Universidad de Alicante \\ Antonio.aledo@ua.es \\ Hugo García \\ Universidad de Alicante \\ Hugo.andreu@ua.es \\ GuAdALUPE ORTIZ \\ Universidad de Alicante \\ Guadalupe.ortiz@ua.es
}

Recibido: 28.01 .2009

Aceptado: 17.05 .2010

\section{INTRODUCCIÓN}

Durante el análisis de los fenómenos sociales los científicos descubren, infelizmente, que éstos tienen orígenes multicausales. Aún más, en el análisis de las causas de los problemas sociales aparecen elementos que adoptan la doble naturaleza de causa/efecto. Así, un factor generador de impacto puede ser, a su vez, producto de una o varias causas (Tufte, 2001). Para mayor dificultad de este ejercicio científico, el propio problema es, como todo fenómeno en el ámbito de lo social, una construcción (Hanningan, 1995).

Desde la Academia se ha destacado la vulnerabilidad de los municipios costeros del Mediterráneo español que han optado por la especialización económica del turismo residencial, tanto desde una perspectiva de cambios en los mercados emisores (Vera y Monforte, 1994; Olcina y Rico, 2005) como de alteraciones en su medio ambiente (Aledo, 2008). También se ha señalado que un parte importante de los problemas que padecen estos municipios son resultado de una rápida y errónea planificación urbanística y turística desarrollada para atender las exigencias de una demanda en constante y rápido crecimiento (MUNRES, 1995; Navalón, 1995; Vera, 1997; Mazón, 2006). Esta interpretación de los 
hechos constituye el discurso social más extendido y políticamente aceptado. Por ejemplo, el Ministerio de Medio Ambiente y Medio Rural y Marino mantiene que «la situación del litoral español responde a una mala planificación urbanística y a un exceso de edificabilidad, con independencia de que la corrupción sea, lamentablemente, una realidad en zonas de la costa.» (diario El País, 15/03/2010). Esta manifestación viene a apoyar la tesis de que el diagnóstico más extendido de los principales impactos negativos que están experimentando los municipios turístico-residenciales del Mediterráneo español, apuntan a factores exógenos o a causas muy superficiales y de carácter técnico, cuando, sin embargo, como se expone en este artículo, puede ser también consecuencia de causas internas y estructurales.

La hipótesis que sustenta esta investigación señala que en los destinos turísticos maduros los impactos no son resultado exclusivamente de fuerzas exógenas. Por el contrario, con el paso del tiempo y su imbricación en lo local, estos impactos terminan transformándose en elementos estructurales y estructurantes del destino turístico. Así, las interacciones entre el turismo residencial y las estructuras y elementos locales son densas y ocasionan una compleja trama de múltiples y mutuas interacciones que pueden ser representadas mediante mapas causales.

El objetivo del artículo es mostrar un método para el análisis de las causas profundas de los impactos inducidos por el turismo residencial en destino maduros. Este método se construye a partir de la elaboración de mapas causales analizados con los programas informáticos de análisis de redes sociales (Ucinet ${ }^{\circledR}$ y NetDraw ${ }^{\circledR)}$ y elaborados en colaboración con los grupos de afectados e interesados locales (a partir de ahora partes interesadas) del turismo residencial.

\section{Los mapas causales}

Para la visualización y el análisis de las complejas estructuras de causa-efecto de cualquier fenómeno social, los científicos han desarrollado el método de los mapas causales. Un mapa causal es una herramienta que permite representar gráficamente las variables y las relaciones causales que tienen lugar en la generación del asunto abordado (Weick, 1979; Gilmore y Murphy, 1991; Weber y Michael, 2001; Miles y Huberman, 1994). Las variables representan las opiniones, ideas y/o temas clave del problema investigado y son representados gráficamente mediante nodos. Estos nodos están conectados mediante flechas unidireccionales que muestran el sentido de la causalidad. Aunque los mapas causales pueden ser construidos a partir de entrevistas individuales, cada vez resulta más habitual elaborarlos mediante reuniones grupales (Ackerman y Eden, 2001). Realizados en el marco de un grupo de trabajo, los mapas causales colectivos representan el punto de vista común que un grupo tiene del asunto analizado una vez se han discutido las diferentes posiciones. Con este método resulta más sencillo que con otras técnicas cualitativas alcanzar acuerdos y consenso entre las 
diferentes posiciones de los participantes (Albino et al., 2002). Los mapas causales son utilizados por los gestores para centrar la atención en las causas de fondo de los problemas (Evans, 2005). Varios autores han utilizado el método de mapas causales en el ámbito del turismo. Woodside (2009) utiliza los mapas causales como herramienta para alcanzar consenso entre los diferentes stakeholders implicados en el turismo de golf. Nash (2006) aplica la Metodología de Redes Causales con el objetivo de identificar las cuestiones claves que preocupan a los diferentes actores públicos y privados implicados en el desarrollo turístico rural de tres destinos escoceses. Una vez elaborados los mapas causales, éstos pueden ser analizados y comparados mediante programas informáticos. Así se ha aplicado a los procesos de toma de decisiones originariamente en el ámbito empresarial, tal y como han hecho Cossette y Audet (1992) y Moretti (1997) empleando el programa informático Decision Explorer ${ }^{\circledR}$.

El análisis de redes sociales (ARS) tradicionalmente ha sido empleado en el estudio de las interacciones entre los actores de una comunidad u organización (Rodríguez, 1995, 2000 y 2004). En el ámbito del turismo destaca el libro de Scott, Baggio y Cooper (2008) donde se aborda el empleo de la ARS en aspectos tales como la planificación pública, la gobernanza, las redes empresariales, el manejo del conocimiento o la estructura de los destinos. Para el caso español cabe mencionar el trabajo de Merinero y Pulido (2009) en el que mediante el ARS demuestran la existencia de correlación positiva entre el desarrollo turístico de un destino y la existencia de densas redes entre actores del sector.

La novedad que presenta este artículo radica en que los mapas causales de impactos inducidos por el turismo residencial son analizados con los programas informáticos desarrollados por el ARS para el análisis de actores. Este método permite gestionar un gran volumen de información relacional e identificar las causas de mayor repercusión sobre las estructuras ambiental, económica y social de los municipios turísticos. Al señalar las causas más influyentes en el sistema turístico, se ofrece una herramienta que ayuda al planificador local a seleccionar las actuaciones más rentables en términos de inversión de recursos.

\section{Mapas causales y estrategias colaborativas}

Como ha sido mencionado, los mapas causales pueden ser elaborados mediante una estrategia colaborativa en la que participan las diferentes partes interesadas en el turismo residencial a nivel local. La selección de esta estrategia se fundamenta en que al interactuar el turismo residencial con las estructuras locales aparecen múltiples interesados y afectados (Keogh, 1990). La apertura del ámbito de la planificación y gestión turística a la participación de nuevos agentes sociales ha sido defendida por muchos expertos en estudios turísticos. En este sentido Murphy enfatiza en la importancia de las estrategias de planificación que incorporan los objetivos y deseos de la comunidad local. Las razones expuestas por este autor aluden a que el desarrollo turístico debe servir tanto a turistas 
como a residentes locales ya que estos últimos son parte del producto turístico y que su exclusión puede derivar en acciones de resistencia y hostilidad que pueden «destruir el potencial total de la industria» (Murphy, 1985: 153). En esta línea, Long (1993) y Gunn (1994) señalan que si la población local afectada no es incorporada en el proceso de planificación, hasta el mejor documento de planificación puede resultar un fracaso. De forma similar, Ap (1992) y Lankford (1994) señalan la importancia de tener en cuenta las percepciones de los residentes de los impactos del turismo para aumentar las posibilidades de éxito en la planificación y políticas turísticas (Lane, 1994; Hemmati, 2002). Del mismo modo, otros autores (Jamal y Getz, 1995; Brohman, 1996; Mazón et al., 1996; Tosun y Timothy, 2003) argumentan que la participación de la población local resulta positiva al redundar en un aumento de la información de base sobre la que se apoyan las decisiones posteriores. Asimismo, esta estrategia favorece la producción de consensos a la hora de identificar las causas de fondo de los problemas locales y, al mismo tiempo, se genera información de calidad que permite mejorar la toma de decisiones durante el diseño de opciones estratégicas para el desarrollo local (Ortiz et al, 2007). Por su parte Bramwell y Sharman (1999) destacan algunos inconvenientes del empleo de la estrategia colaborativa, destacando la influencia que puede tener en el proceso de diagnóstico la desigual posición de poder de las diferentes partes interesadas participantes. La investigación que se presenta a continuación ha pretendido abordar estas dificultades a través de dos estrategias fundamentales: por un lado, el desarrollo de un análisis de las partes interesadas que considera la variable poder entre los criterios de estudio (Mitchell et al, 1997) ha permitido asegurar la correcta selección de los mismos mediante la atención no sólo de las estructuras de poder intergrupales sino también intragrupales (Winn, 2001). Por otro lado, este obstáculo se afronta también mediante la adopción de un enfoque deliberativo, en el que no ha de primar tanto la posición estructural de los participantes como «la fuerza del mejor argumento» (Manin, 1987; Elster, 1998), enfatizándose así la situación de igualdad que adquieren los participantes durante el hecho deliberativo (Nino, 1996; Cohen, 1997).

\section{Los impactos del turismo residencial}

A partir de los desarrollos teóricos sobre impactos sociales de Vanclay (2000), Becker y Vanclay (2003) y Burdge (2004), esta investigación ha construido una definición ad hoc de impactos del turismo residencial entendidos como los efectos inducidos por esta actividad sobre las estructuras locales. Estos impactos han sido identificados a partir de la percepción de las partes interesadas (Stolp, 2003). Para su análisis, estas estructuras locales se han agrupado en ambientales, económicas, socioculturales e infraestructuras y servicios públicos. De este modo, a lo largo de la investigación se identificaron como impactos de la actividad turístico residencial el déficit en la oferta de servicios sanitarios, la de- 
gradación del paisaje agrícola, el incremento de los precios o la segregación espacial entre grupos de diferentes nacionalidades. Seguidamente, antes de presentar la propuesta metodológica se lleva a cabo una revisión de la literatura científica existente sobre los impactos del turismo residencial y se describe el caso de estudio.

El turismo residencial ha recibido por parte de la turismología cierta atención en los últimos años. La bibliografía española y latinoamericana (Ferreira y Cardoso, 2007; Da Silva y Ferreira, 2010) ha aceptado el término turismo residencial indagando en su naturaleza (Mazón, 2006), impactos (Cañada, 2010) y en los tipos de demanda (Monreal, 2001). Por su parte la bibliografía anglosajona ha mostrado notables reticencias a la denominación de turismo residencial (Hall, 2004) y ha preferido el término second homes (Coppock, 1977) o bien ha orientado sus investigaciones a las Migraciones Internacionales de Retirados (Williams, 1997; Rodríguez et al., 1998; King, 2000; Warnes, 2001), conceptualizadas por O'Reilly como como lifestyle migrations (Benson, 2009). No obstante, la línea anglosajona parece comenzar a aceptar el término y así en un reciente trabajo McWatters ha definido el turismo residencial desde una aproximación amplia como «las prácticas y estilos de vida duraderos que se derivan de flujos canalizados de migración permanente o semipermanente a un destino concreto» (2009: 3).

En España, las investigaciones sobre impactos del turismo residencial se han orientado principalmente a su identificación y caracterización (Torres, 2003; Exceltur, 2005; Garcia-Andreu, 2005; Mantecón, 2005). Los especialistas en la materia ha señalado los notables impactos ambientales del turismo residencial sobre los ecosistemas; tales como la degradación del paisaje, reducción de biodiversidad, deforestación e incremento de los incendios forestales, pérdida de la capa vegetal, erosión y desertificación, y el incremento de la contaminación edáfica, acústica y de las aguas superficiales y subterráneas (Garnert, 1987; Grenon, 1990; Almenar, 2000; OSEab, 2006; Greenpeace, 2007). Por su parte los especialistas en demografía que han analizado el impacto del turismo residencial sobre las comunidades locales han destacado el envejecimiento de la población y, por ende, el aumento de las tasas de dependencia, la llegada de inmigrantes residenciales y laborales y el rápido aumento demográfico experimentado por estas localidades en cortos espacios de tiempo (Casado, 1999). Otros investigadores han destacado impactos económicos positivos como el aumento de las rentas y la generación de empleo (Ros, 2003). No obstante, se ha señalado el riesgo de optar a nivel local por la monofuncionalidad del turismo residencial y la dependencia de las haciendas locales de los ingresos por la actividad inmobiliaria y constructiva asociada a esta actividad (Arrocha, 2005). Entre los impactos socioculturales más destacados se ha citado el abandono de actividades tradicionales, procesos de segregación urbana (Aledo, 2008), y conflictos latentes entre grupos de diferentes nacionalidades que luchan por recursos escasos, conflictos idiomáticos y otros relacionados con los cambios en la identidad local (Nogues-Pedregal, 2010). Por último, varios autores han ligado el rápido creci- 
miento del turismo residencial con graves procesos de corrupción a escala municipal (Díez Ripollés et al., 2003; Greenpeace, 2006; Transparency International, 2006; Diez y Gómez-Céspedes, 2008; Jiménez, 2008).

La información de caso con la que se ejemplifica esta propuesta metodológica se ha obtenido de un proyecto de investigación ${ }^{1}$ desarrollado durante los años 2006 y 2007 en el municipio de Dénia ubicado en el norte de la Costa Blanca (provincia de Alicante). Esta investigación presentaba como objetivo principal el diseño de propuestas para la reorientación de municipios turístico maduros de la provincia de Alicante con un alto grado de especialización en el turismo residencial.

\section{EL CASO DE DÉNIA}

Diversos estudios han señalado que Dénia se encuentra en una fase madura de desarrollo turístico-residencial (Mazón y Huete, 2005; Aledo y Mazón, 2005). Sus $20 \mathrm{kms}$. de playas, junto con el Parque Natural del macizo del Montgó y la zona de huerta que rodea el casco urbano, constituyen el escenario turístico y contribuyen a explicar su rápido desarrollo turístico-residencial iniciado a mitad de la década de los sesenta del siglo pasado. En esos años comienza a abandonarse las actividades tradicionales de la agricultura, pesca y la industria del juguete para volcarse en el sector inmobiliario y en los servicios asociados al turismo (Vera, 1992; Ciudad, 1995). El crecimiento es tal que los 12.185 habitantes registrados en 1960 se doblan en 1991, para superar los 40.000 quince años más tarde (INE, 2008). Este crecimiento se debe a los fuertes movimientos inmigratorios, primero de españoles del centro y sur de la Península atraídos por la demanda de mano de obra que genera el turismo y la construcción y, segundo, de jubilados europeos (García-Andreu y Rodes, 2004), al que a partir de 1999, se le suman inmigrantes laborales procedente de países en desarrollo (Viruela y Domingo, 2001). Tras cuarenta años de turismo residencial, su estructura de alojamiento turístico está fuertemente desequilibrada. En 2001 con datos del Censo de Población y Vivienda, Dénia ofertaba 78.299 plazas extra-hoteleras (GarcíaAndreu, 2008: 202) frente a tan sólo 1.321 plazas hoteleras. Este desarrollo ocasiona que el sector turístico de Dénia muestre todas las características del turismo residencial en su etapa central (Aledo, 2008), a saber, fuerte estacionalidad y concentración espacial, crecimiento urbanístico espontáneo y atomizado, una oferta complementaria poco desarrollada, un fuerte impacto ambiental y paisajístico y una excesiva dependencia del sector de la construcción (Mazón, 2006). A mediados de los noventa el informe provincial MUNRES (1995) aconsejaba tomar una serie de medidas para reorientar el desarrollo turístico-residencial en

${ }^{1}$ Proyecto de I+D+i financiado por el Ministerio de Educación y Ciencia con título «Turismo residencial: análisis de la demanda y propuestas de reestructuración para los destinos consolidados» SEJ/2005/04305. 
este tipo de municipios. A las recomendaciones de los expertos se unen los resultados de una Encuesta Socioambiental ${ }^{2}$ realizada en el municipio de Dénia en enero de 2003. El 65,6\% de los encuestados consideraban la construcción de urbanizaciones como una actividad perjudicial y 3 de cada 4 ciudadanos consultados opinaban que el ritmo de construcción en Dénia debía reducirse. Los efectos más negativos asociados a la actividad inmobiliaria eran problemas de tráfico $(92,4 \%)$ reducción de la disponibilidad de agua $(75,5 \%)$ y ausencia de equipamientos sociales y sanitarios $(75,6 \%)$. En definitiva, los datos revelaban un amplio acuerdo social en la necesidad de reconducir el modelo de desarrollo local.

\section{PROPUESTA DE DIAGNÓSTICO DE LOS IMPACTOS LOCALES DEL TURISMO RESIDENCIAL}

Tal y como se ha señalado, los mapas causales son elaborados mediante una estrategia colaborativa en la que participan las diferentes partes interesadas en el turismo residencial a nivel local. Su intervención tiene lugar en las dos primeras fases de análisis de los impactos; a saber, identificación de los impactos a escala local y elaboración de los mapas causales de dichos impactos. La tercera fase de esta propuesta la constituye el análisis informático de redes del conjunto de mapas causales.

\section{Primera fase: Identificación de los impactos a escala local}

El objetivo principal de esta fase consiste en identificar los impactos inducidos por el turismo residencial sobre las estructuras ambiental, económica y social del municipio objeto de estudio. En primer lugar se identifican las diferentes partes interesadas en el turismo residencial a nivel local. En el caso de Dénia se reconocieron las siguientes: (1) turistas residenciales españoles, (2) retirados internacionales, (3) población local de nacionalidad española, (4) inmigrantes laborales extranjeros, (5) empresarios locales, (6) políticos locales, (7) técnicos municipales y (8) profesionales de los medios de comunicación. Su selección se fundamentó en que al ser actores que arriesgan algo en el turismo residencial local, procurarán un buen nivel en la calidad de la información a manejar (Funtowicz y Ravetz, 2000). Así, cada una de las partes interesadas seleccionadas aportan información de calidad desde dentro, desde su experiencia como co-protagonistas de un fenómeno social que, además de envolverlos, contribuyen en diferente medida a construir. Los representantes de las partes interesadas fueron seleccionados, en unos casos, siguiendo la técnica «bola de nieve» realizada desde

${ }^{2}$ Elaborada por el Departamento de Sociología 1 de la Universidad de Alicante a petición de la Concejalía de Medio Ambiente de Dénia (dir. Antonio Aledo).

EMPIRIA. Revista de Metodología de Ciencias Sociales. N. ${ }^{\circ}$ 20, julio-diciembre, 2010, pp. 61-86. ISSN: 1139-5737 
las primeras fases de la investigación y, en otros, acudiendo a los gerentes de las asociaciones e instituciones. En segundo lugar, mediante entrevistas individuales semiestructuradas y grupos de discusión, se recaba la opinión de las partes interesadas sobre los impactos del turismo residencial. En el caso de Dénia participaron en esta fase 48 representantes. En tercer lugar, con esta información, se elabora un listado común que identifica los efectos inducidos por el turismo residencial. En el caso de estudio se identificaron 66 impactos, listados en un documento que constituye el documento de partida de la segunda fase.

\section{Segunda fase: Mapas causales de los impactos}

El objetivo específico de esta segunda fase consiste en identificar los mapas causales que generan los impactos recabados en la fase anterior. En el caso de Dénia se organizó un taller al que aceptaron participar 25 representantes de las partes interesadas que se repartieron en cuatro mesas de trabajo, a saber: Infraestructuras y servicios, Medio ambiente, Economía y Sociedad. Por razones de dinámica de trabajo y de agenda no se pudo trabajar con la totalidad de impactos identificados. Así, de los 66 impactos, los participantes priorizaron 16 (a partir de ahora impactos $_{p}$ ), para trabajar cuatro en cada grupo (cuadro 1).

Cuadro 1. Impactos $_{p}$ seleccionados.

\begin{tabular}{|c|c|}
\hline \multirow{4}{*}{ Área temática } & Impactos seleccionados \\
\hline \multirow{4}{*}{ Infraestructuras y servicios } & Déficit de aparcamiento \\
\cline { 2 - 2 } & Déficit de infraestructuras viarias \\
\cline { 2 - 2 } Medio ambiente & Déficit de servicios sanitarios \\
\cline { 2 - 2 } & Insuficiente oferta complementaria (ocio, deporte, \\
comercio y cultura)
\end{tabular}

Fuente: Elaboración propia. 
Mediante un proceso reflexivo-deliberativo, los participantes representan en mapas causales (Ver figura 1) los elementos generadores de los impactos ${ }_{p}$ (ver Glosario). Estos elementos se denominan a partir de ahora nodos. El esfuerzo participativo y deliberativo favorece que los mapas causales construidos no se limiten a recoger las causas más evidentes y generales que producen los impac$t_{0}$. Por el contrario, dicha deliberación hace emerger los elementos causales de mayor capacidad explicativa (Jamal y Getz, 1995; Brohman, 1996; Tosun y Timothy, 2003). Así, cada mapa causal, de forma individual, aporta información sobre las causas de un nivel más profundo que motivan un determinado impacto detectado en la fase de diagnóstico. De esta manera se evitan actuaciones «superficiales» en la planificación, que supongan una intervención sobre los síntomas antes que sobre las causas «profundas» de los mismos. Los participantes identificaron 209 nodos para el conjunto de los 16 mapas causales construidos.

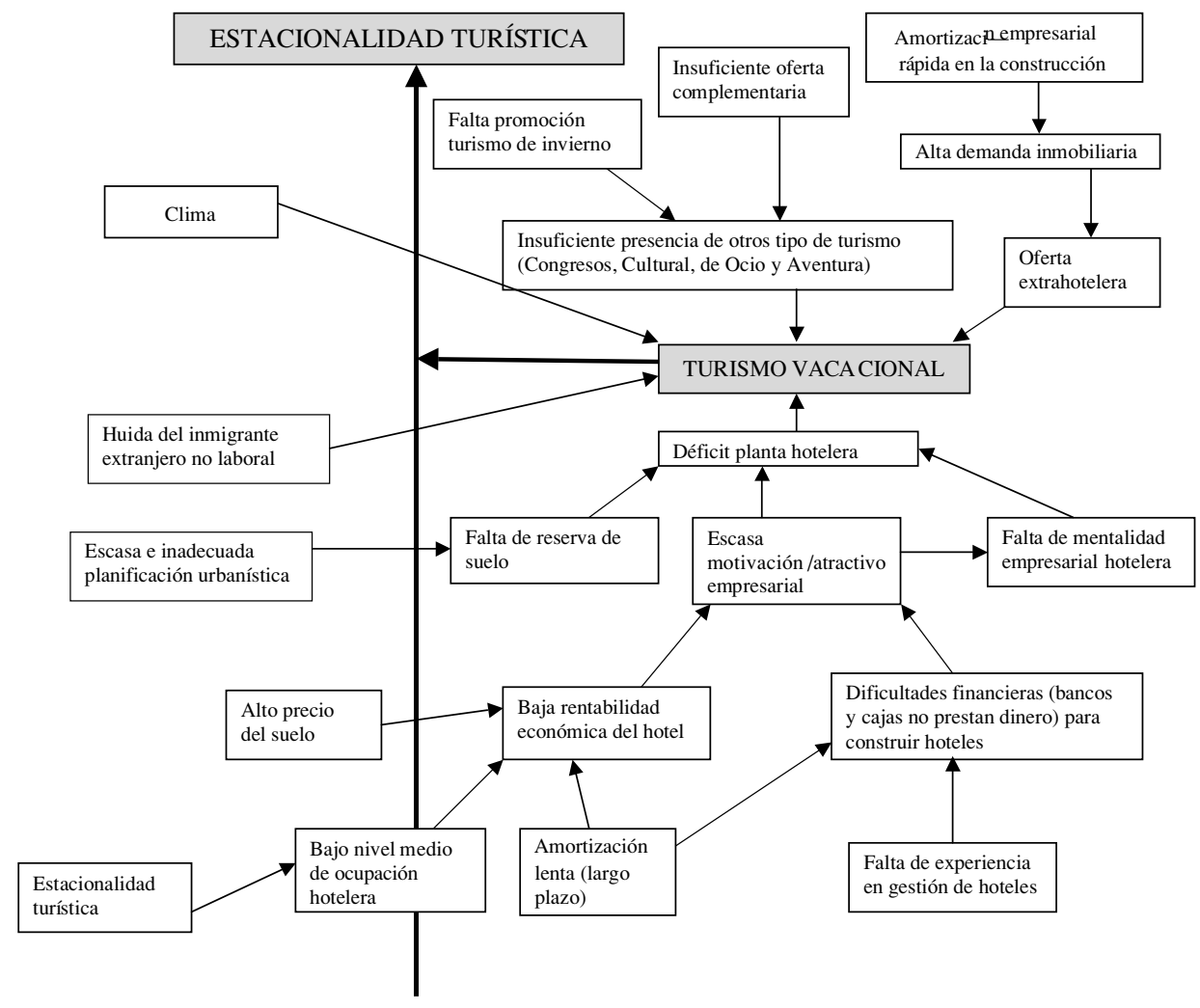

Fuente: Elaboración propia.

Figura 1. Mapa causal del impactop Estacionalidad turística. 
Así, los mapas causales elaborados son útiles para mejorar el proceso de diagnóstico. No obstante, el análisis aislado e individual de los diferentes mapas causales no permite trazar y mostrar el orden complejo de flujos de repercusión inducido por los impactos locales del desarrollo turístico (Mathieson y Wall, 1990). En otras palabras, los impactos del turismo y sus causas y consecuencias no se sitúan en la realidad en cajas aisladas. Por el contrario, conforman una intrincada red de relaciones directas e indirectas que es necesario analizar desde una perspectiva estructural y holística.

\section{Tercera fase: El análisis estructural de los impactos}

Con el objeto de solventar la limitación señalada, se incorpora al proceso de diagnóstico de los impactos locales del turismo residencial el análisis de redes de los mapas causales mediante el uso de los programas informáticos $U c i$ net ${ }^{\circledR}$ y NetDraw ${ }^{\circledR}$. Este tipo de análisis permite 1) incorporar de forma sistemática las relaciones directas e indirectas entre los nodos del sistema, 2) elaborar un mapa estructural de impactos inducidos por el turismo residencial y 3) identificar así las causas con mayor influencia en dicha estructura relacional.

De forma previa al análisis informático de redes, se llevan a cabo dos acciones sucesivas: a) la agregación de los nodos presentes en los mapas causales y b) la elaboración de una matriz relacional.

\section{a) Agregación de nodos}

El primer paso para tratar la información contenida en los mapas causales consiste en desarrollar un proceso de agregación o reducción. Esto es, los nodos que en los mapas causales presentan significados similares pero con una terminología diferente, son sustituidas por una categoría nominal común. Así, en el proceso de elaboración de la matriz relacional, se reduce el número de nodos, pero aumenta el número de relaciones de algunas de ellas. En el caso de estudio, el resultado de este proceso redujo el número de nodos con el que elaborar la matriz de 209 a 197. Por ejemplo, en la figura 1, aparece el mapa causal del impacto Estacionalidad turística. Una de sus causas es la Escasa e inadecuada planificación urbanística. Este nodo es una categoría nominal común establecida para sustituir términos como el de Mala planificación o Falta de planificación aparecidos en la elaboración de algunos mapas causales.

\section{b) Elaboración de la matriz relacional}

Esta acción supone convertir las relaciones causa-efecto presentes en los mapas causales en una matriz relacional que tiene las siguientes características: 
a. Es una matriz cuadrada, por lo que tiene los mismos nodos (197) en las filas y en las columnas.

b. Es una matriz asimétrica, de tal modo que la existencia de influencia de A sobre B no implica la existencia de influencia inversa (de B hacia A); es decir, las influencias son unidireccionales.

\section{ANÁLISIS INFORMÁTICO DE REDES}

La matriz relacional constituye la base de datos con la que operan los programas de análisis de redes. NetDraw ${ }^{\circledR}$ produce una representación visual en forma de red de la matriz de datos elaborada (Ver figura 2).

La representación visual de la red sitúa en un plano los nodos analizados y muestra las relaciones que mantienen con otros nodos. Por su parte, el análisis estadístico (Ucinet $\left.{ }^{\circledR}\right)$ permite calcular de forma sistemática el número de relaciones directas e indirectas que mantiene cada uno de los nodos en el conjunto de la red y, así, establecer su centralidad. Para comprender las potencialidades de este análisis es conveniente hacer referencia a la importancia de las influencias indirectas.

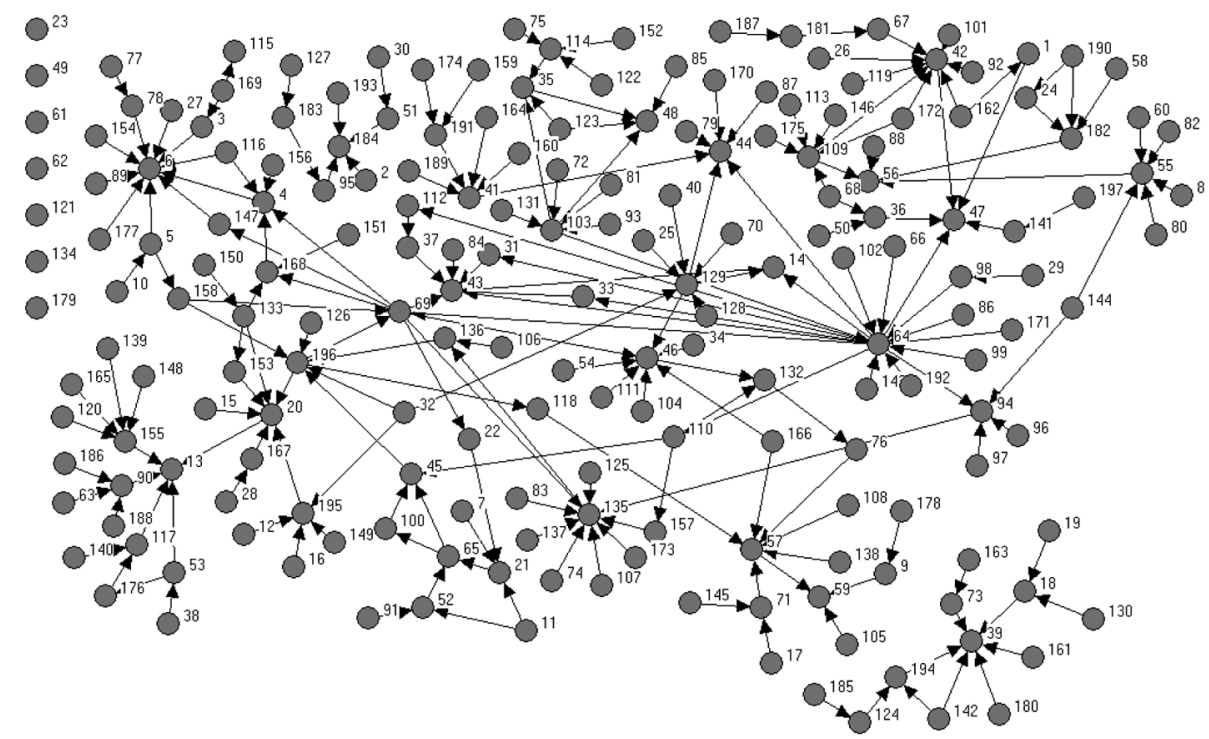

Fuente: Elaboración propia.

Figura 2. Representación visual del mapa estructural de impactos. 


\section{La importancia de las influencias indirectas}

Se entiende por influencia indirecta aquella relación entre dos nodos en donde media, por lo menos, un tercero. Por ejemplo, en la figura 1. la relación entre Escasa e inadecuada planificación urbanística y Déficit planta hotelera es indirecta al estar mediada por el nodo Falta de reserva de suelo. Dada la interconexión entre los mapas causales, la correcta apreciación de las influencias indirectas entre los nodos resulta esencial para un correcto análisis de la centralidad de la red de impactos inducidos por el turismo residencial.

Si bien en la imagen de la red (figura 2) están presentes tanto las influencias directas como las indirectas, resulta muy complicado mediante un examen visual realizar un análisis sistemático de las relaciones indirectas entre los nodos, dada la compleja trama de conexiones existente. Quedarse sólo en el análisis de la centralidad de la red partir de las relaciones directas entre los elementos tendría una serie de inconvenientes:

1. Sólo reflejaría una parte del entramado de las relaciones entre los nodos de la red.

2. Sólo se conocerían las influencias que tiene un nodo con los nodos de su entorno inmediato, desconociendo las relaciones que mantiene en el conjunto de la red.

3. La centralidad de la red a partir de las relaciones directas presentaría una elevada sensibilidad a la procedencia de la información con la que se elabora la red. De este modo, si las partes interesadas reunidas para elaborar un determinado mapa causal han aportado un elevado número de causas para explicar el impacto $_{p}$, condicionarán la centralidad de la red.

Así, el análisis exclusivo de las relaciones directas supone obviar una parte relevante de la red de impactos. Sin embargo, mediante la inclusión de las relaciones indirectas entre los nodos se consigue solucionar los inconvenientes anteriores, a saber:

1. Manejar la compleja red relacional existente entre el conjunto de nodos de los mapas causales; es decir, este tipo de análisis permite tratar de forma sistemática las relaciones indirectas entre todos los nodos contenidos en cada uno de los mapas causales.

2. Conocer la posición de un nodo en la red total — resultado de la combinación de todos los mapas causales- y no en la red local que muestra un mapa causal o las relaciones más evidentes en la imagen de la red (figura 2).

3. Minimizar la sensibilidad del análisis de la centralidad al proceso de producción de la información (elaboración de mapas causales); en otras palabras, al incluir en el cálculo de la centralidad las relaciones indirectas entre nodos pertenecientes a diferentes mapas causales, se consigue reducir el peso específico de las causas identificadas en un mapa causal. 
Para analizar la centralidad a partir de las influencias indirectas, se utiliza el análisis estadístico (Ucinet $\left.{ }^{\circledR}\right)$ de redes de la matriz relacional, en concreto se emplea el indicador estadístico de la cercanía.

\section{Análisis de las influencias indirectas: el indicador estadístico de Cercanía}

El indicador estadístico de la cercanía mide la distancia media de cada nodo con respecto al resto de nodos de la red. Este indicador tiene en cuenta las relaciones indirectas entre los diferentes nodos. Así, son más centrales, de modo global en la red, los nodos con un mayor índice de cercanía o, en otras palabras, aquéllos que tienen que dar un menor número de pasos para relacionarse con (e influir en) el mayor número de nodos de la red (Rodríguez, 1995). En este caso, al ser una matriz asimétrica (influencias unidireccionales entre los elementos) obtenemos de forma diferenciada la cercanía de las influencias ejercidas y la cercanía de las influencias recibidas.

\section{Análisis de las influencias indirectas recibidas: Cercanía recibida}

Para comprobar los cambios en la centralidad de la red al introducir en el análisis las relaciones indirectas entre los nodos, resulta interesante comparar el indicador de cercanía con el de grado. Este último indicador estadístico, a diferencia del anterior, mide las relaciones directas entre los nodos. Al igual que el indicador de cercanía, al ser una matriz asimétrica nos ofrece tanto las influencias directas ejercidas como las recibidas. A continuación, empleando la información obtenida en el caso de estudio, se evidencia las diferencias existentes al comparar el ranking que resulta de analizar las influencias que los 197 nodos de la matriz reciben de forma directa ( $\mathrm{grado}$ ) con las influencias que reciben de forma indirecta (cercanía) (Tablas 1a y 1b).

Dado que el objetivo que preside esta tercera fase es localizar de forma sistemática los nodos más centrales, las tablas anteriores sólo muestran aquéllos que se encuentran por encima de la media del rango de valores posibles de dicho indicador. Por ejemplo, en el indicador de las influencias directas recibidas (tabla 1a), el rango de valores va de cero a diez. Por lo tanto se han discriminado todos los nodos que presentan un valor igual o inferior a la media (5). De este modo, sólo diez nodos superan la criba (el 5\% del total). Sin embargo, cuando se observa la tabla que muestra el indicador de las influencias indirectas recibidas (tabla 1b), aparecen veinte nodos que se encuentran por encima del valor medio del rango, el 10\% del total. Es decir, al incluir las relaciones indirectas en el análisis de la red se producen importantes modificaciones en la tabla. Por un lado, se duplica el número de nodos con una elevada accesibilidad en términos de influencias y, por otro lado, varía su orden. En la tabla 1a se resaltan en cursiva aquellos nodos que desaparecen en la tabla $1 \mathrm{~b}$ debido a que, al tener en cuenta en esta última el indicador de cercanía, su posición en el ranking desciende por de- 
Tablas 1a y 1b. Comparativa entre las influencias recibidas de forma directa (grado) e indirecta (cercanía)

Tabla a. Influencias directas recibidas

\begin{tabular}{ccc}
\hline $\begin{array}{c}\text { Influencias } \\
\text { directas recibidas } \\
\text { (Indegree) }\end{array}$ & $\begin{array}{c}\text { Etiqueta } \\
\text { en la red }\end{array}$ & Nodos \\
\hline 10 & 6 & Alto precio de la vida \\
\hline 9 & 64 & $\begin{array}{c}\text { Escasa e inadecuada planificación urbanística } \\
\text { Insuficiente oferta complementarria }\end{array}$ \\
\hline 8 & 135 & Crisis económica del sector agrario \\
\hline 7 & 42 & Déficit de aparcamientos \\
& 43 & Déficit serviciois sanitarios \\
\hline & 46 & Aumento población \\
& 20 & Contaminación acústica \\
& 39 & Déficit infractructuras viarias \\
& 44 & El ayuntamiento gasta más que ingresa \\
\hline
\end{tabular}

Tabla 1b. Influencias indirectas recibidas

\begin{tabular}{|c|c|c|}
\hline $\begin{array}{c}\text { Influencias } \\
\text { indirectas recibidas } \\
\text { (Incloseness) }\end{array}$ & $\begin{array}{l}\text { Etiqueta } \\
\text { en la red }\end{array}$ & Nodos \\
\hline 0,787 & 13 & Aumento consumo agua \\
\hline 0,471 & 6 & Alto precio de lla vida \\
\hline 0,723 & 59 & Endeudamiento municipal \\
\hline 0,711 & $\begin{array}{l}43 \\
46\end{array}$ & $\begin{array}{c}\text { Déficit aparcamientos } \\
\text { Déficit servicios sanitarios }\end{array}$ \\
\hline 0,705 & 20 & Aumento población \\
\hline 0,704 & 57 & El Ayuntamiento gana más que ingresa \\
\hline 0,686 & 4 & Alquileres altos en vivienda \\
\hline 0,672 & 168 & Poca ofrta alquiler vivienda \\
\hline \multirow{2}{*}{0,659} & 147 & $\begin{array}{l}\text { Más demanda que oferta en temporada y luego } \\
\text { se mantiene los precios }\end{array}$ \\
\hline & 118 & $\begin{array}{l}\text { Gastos estacionales en servicios y } \\
\text { mantenimiento }\end{array}$ \\
\hline 0,656 & $\begin{array}{c}69 \\
135 \\
196\end{array}$ & $\begin{array}{l}\text { Estacionalidad turística } \\
\text { Insuficiento oferta complementaria } \\
\text { Turismo vacacional }\end{array}$ \\
\hline 0,655 & $\begin{array}{c}12 \\
136\end{array}$ & $\begin{array}{l}\text { Bajo nivel medio de ocupación hostelera } \\
\text { Insuficiente presencia otro tipo turismo }\end{array}$ \\
\hline 0,654 & $\begin{array}{l}21 \\
45 \\
65\end{array}$ & $\begin{array}{c}\text { Baja rentabilidad económica del hotel } \\
\text { Déficit planta hotelera } \\
\text { Escasa motivación, atractivo empresarial por el } \\
\text { negocio hotelero }\end{array}$ \\
\hline 0,653 & 100 & Falta mentalidad empresarial hotelera \\
\hline
\end{tabular}

Fuente: Elaboración propia.

EMPIRIA. Revista de Metodología de Ciencias Sociales. N. ${ }^{\circ}$ 20, julio-diciembre, 2010, pp. 61-86.

ISSN: $1139-5737$ 
bajo del valor de corte. Un ejemplo de lo señalado lo constituye el nodo Escasa e inadecuada planificación urbanística (identificada con el número [64] en la imagen de la red - figura 2) que, obteniendo una de las puntuaciones más altas cuando se analizan las influencias directas (9), desaparece de la tabla para dejar paso a otros nodos más conectados en términos de influencias indirectas. Este es el caso de los nodos destacadas en cursiva de la tabla $1 \mathrm{~b} \mathrm{y}$, de forma especial, de Aumento en el consumo de agua [13]. Este nodo, sin presencia en la tabla 1a (por recibir 5 influencias), alcanza el primer puesto al introducir en el análisis las relaciones indirectas.

En definitiva, en lo que respecta a la cercanía recibida de los nodos el análisis sistemático y sistémico de las relaciones indirectas ponen de manifiesto el complejo entramado de repercusiones. Así se demuestra que cada una de los nodos está más conectado con el resto de lo que parece al analizar las relaciones directas. De este modo se puede observar el papel que juegan los nodos en la red total. Así, el análisis de la centralidad se muestra menos sensible a las causas identificadas durante el proceso de elaboración de un mapa causal concreto, dándose más peso a la posición que ocupa el nodo en la red total. Por ejemplo en el caso de estudio, el nodo Aumento en el consumo de agua [13], que no aparece en la tabla 1a, ocupa el primer puesto en la tabla que recoge las influencias indirectas recibidas (tabla $1 \mathrm{~b}$ ).

Continuando con el análisis de los valores obtenidos en el indicador de cercanía recibida, éstos se pueden interpretar como una medida de la probabilidad que tienen los impactos de verse afectados ante una actuación sobre los nodos de la red. Un buen ejemplo en este sentido se encuentra en el impacto Aumento $_{p}$ en el consumo de agua [13] (Tablas 1a y 1b). Su elevada cercanía recibida (la mayor del mapa estructural de impactos) implica que existe un buen número de nodos que lo definen y que facilitan su modificación. Estos nodos pueden pertenecer a su entorno más cercano, identificados en su mayoría en la elaboración del mapa causal, ya que dicho nodo [13] es un impacto. . Estos nodos son fácilmente localizables en la imagen de la red (figura 2). Por ejemplo, en el caso del

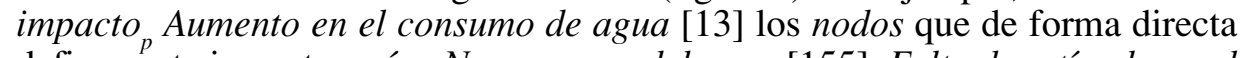
definen este impacto serían Nuevos usos del agua [155], Falta de estímulo en el ahorro [90], Fugas en la red de distribución de agua [117], Diseño horizontal de la ciudad [53] y Aumento de población [20], así como los nodos que a su vez definen y concretan a éstos. En el caso del nodo Fugas en la red de distribución de agua [117], éstas serían Mal estado de las tuberías [140] y Red de tuberías expansivas [176].

No obstante, la elevada accesibilidad de Aumento en el consumo de agua [13] también se explica porque existen otros nodos más distantes en términos relacionales y, por tanto, menos evidentes en la imagen de la red, pero que igualmente definen el problema y constituyen una puerta de entrada a su modificación. Los programas informáticos Ucinet ${ }^{\circledR}$ y NetDraw ${ }^{\circledR}$ no ofrecen de forma sistemática los nodos concretos que de forma indirecta afectan a un elemento del mapa estructural de impactos. Esto sería especialmente útil con las relaciones 
que se encuentran mediadas por otros nodos y que aparecen distantes en la red, ya que su identificación se vuelve muy laboriosa. Aunque costosa, esta tarea se puede realizar recorriendo de forma inversa las influencias que recibe un nodo determinado. No obstante, no todos los eslabones del mapa causal identificados a lo largo de este afanoso proceso constituirán, a pesar de su función de causa, nodos sobre los que resulta factible actuar. La razón es que se encuentran en la zona media del mapa causal y tienen, al igual que los impactos, , nodos por debajo de ellos que los concretan, operativizan y que tienen una mayor radiación en la red. Son estos últimos los nodos que resultan relevantes de cara al análisis de la centralidad de la red, independientemente si para ello se han esclarecido los itinerarios seguidos.

Para identificar de forma sistemática estos nodos se emplea el análisis estadístico ofrecido por el programa informático Ucinet $\AA$. El resultado de dicho análisis no establece los itinerarios concretos de conexión entre los nodos más influyentes de la red de impactos con los efectos finales (impactos $)$. No obstante, al ser estos últimos muy influenciables y aquéllos muy influyentes, se asegura que una actuación sobre los nodos más influyentes redundará en una modificación de los efectos más sensibles y accesibles (impactos $)$. Este análisis estadístico se realiza a partir del examen de las influencias indirectas ejercidas (cercanía ejercida) por los nodos de la red.

\section{Análisis de las influencias indirectas ejercidas: Cercanía ejercida}

Como se acaba de mencionar, el análisis de las influencias indirectas ejercidas permite la identificación de los nodos más influyentes de la red de impactos. $\mathrm{Al}$ igual que con el indicador de cercanía recibida, el indicador de cercanía ejercida muestra los nodos que se encuentran por encima de la media del rango de valores posibles de dicho indicador. Esto se traduce en que en el caso de las influencias directas ejercidas sólo dos nodos superan el corte debido a las causas identificadas en los mapas causales. Sin embargo, al tener en cuenta las influencias indirectas ejercidas (tabla $2 \mathrm{~b}$ ), en la mitad superior del rango de valores se encuentran 46 nodos (el 23\% del total). Es decir, el análisis de las influencias indirectas convierte en centrales 44 nodos (resaltados en cursiva) que, en el análisis de las influencias directas, no tendríamos en cuenta. Así, se comprueba de nuevo que introducir en el análisis las influencias indirectas permite conocer la posición de los nodos en la red total y no únicamente en su entorno relacional más cercano; un entorno relacional que además se encuentra altamente condicionado por las influencias directas imputables a las relaciones de causalidad identificadas en un mapa causal. 
Tablas 2a y 2b. Comparativa entre las influencias ejercidas de forma directa (grado) e indirecta (cercanía).

Tabla 2a. Influencias directas ejercidas.

\begin{tabular}{ccc}
\hline $\begin{array}{c}\text { Influencias } \\
\text { directas ejercidas } \\
\text { (Outdegree) }\end{array}$ & $\begin{array}{c}\text { Etiqueta } \\
\text { en la red }\end{array}$ & Nodos \\
\hline 10 & 64 & Escasa e inadecuada planificación urbanística \\
\hline 7 & 69 & Estacionalidad en la afluencia de turistas \\
\hline
\end{tabular}

Tabla 2b. Influencias indirectas ejercidas.

\begin{tabular}{|c|c|c|}
\hline $\begin{array}{l}\text { Influencias } \\
\text { indirectas ejercidas } \\
\text { (Outcloseness) }\end{array}$ & $\begin{array}{l}\text { Etiqueta } \\
\text { en la red }\end{array}$ & Nodos \\
\hline 0,611 & $\begin{array}{l}72 \\
81 \\
93 \\
131\end{array}$ & $\begin{array}{c}\text { Falta asociacionismo } \\
\text { Falta comunicación entre Ayuntamiento y población } \\
\text { Falta de información del Ayuntamiento a la población } \\
\text { Individualismo histórico }\end{array}$ \\
\hline 0,608 & 103 & Estacionalidad en la afluencia de turistas \\
\hline 0,604 & 29 & Carácter de Dénia: rechazo a los líderes políticos \\
\hline 0,601 & $\begin{array}{c}66 \\
86 \\
99 \\
102 \\
98 \\
143 \\
171 \\
192\end{array}$ & $\begin{array}{c}\text { Escasa presión en el ámbito urbanístico de los } \\
\text { empresarios del comercio } \\
\text { Falta de continuidad política } \\
\text { Falta de mayoría política } \\
\text { Falta de objetivos de carácter urbanístico } \\
\text { Falta liderazgo político } \\
\text { Mala gestión urbanística delos gobiernos locales } \\
\text { Predominio iniciativa privada en la planificación } \\
\text { urbanística } \\
\text { Transferencia de las competencias de las cámaras } \\
\text { agrarias al consejo local agrario }\end{array}$ \\
\hline 0,598 & 64 & Escasa e inadecuada planificación urbanística \\
\hline 0,577 & 32 & Clima \\
\hline 0,573 & 144 & Mala gestión económica por parte de los políticos \\
\hline 0,57 & 10 & $\begin{array}{c}\text { Amortización empresarial rápida en la construcción de } \\
\text { viviendas }\end{array}$ \\
\hline 0,567 & $\begin{array}{c}110 \\
5 \\
11 \\
91 \\
\\
96\end{array}$ & $\begin{array}{c}\text { Falta reserva suelo en la planificación urbanística } \\
\text { Alta demanda inmobiliaria } \\
\text { Amortización empresarial lenta en el negocio hotelero } \\
\text { Falta experiencia gestión hotelera } \\
\text { Falta interés de la administración a la hora de dotar de } \\
\text { infractructuras deportivas, infantiles, culturales, ocio y } \\
\text { comercio } \\
\text { Falta interés empresariosa a la hora de dotar de } \\
\text { infraestructuras deportivas, infantiles, culturales, ocio y } \\
\text { comercio }\end{array}$ \\
\hline
\end{tabular}

EMPIRIA. Revista de Metodología de Ciencias Sociales. N. ${ }^{\circ}$ 20, julio-diciembre, 2010, pp. 61-86. 
Tabla 2b. Influencias indirectas ejercidas (Continuación).

\begin{tabular}{ccc}
\hline $\begin{array}{c}\text { Influencias } \\
\text { indirectas ejercidas } \\
\text { (Outcloseness) }\end{array}$ & $\begin{array}{c}\text { Etiqueta } \\
\text { en la red }\end{array}$ & Nodos \\
\hline & 126 & Huida inmigrante extranjero no laboral \\
Oferta extrahotelera
\end{tabular}

Fuente: Elaboración propia.

A continuación se muestra con un ejemplo la importancia de recoger en el análisis las influencias indirectas ejercidas. Si se observa la posición que ocupan en la imagen de la red los nodos que obtienen la mayor puntuación (Ver figura 2elementos Falta asociacionismo [72], Falta comunicación entre Ayuntamiento y población [81], Falta de información del Ayuntamiento a la población [93] e Individualismo histórico [131]) se comprueba que son nodos con una sola influencia directa ejercida. Pero el hecho de que estos nodos ejerzan su influencia sobre un nodo altamente conectado (Falta de participación [103]) les proporciona la mayor capacidad de influencia sobre la red con el menor número de pasos. Este nodo [103] obtiene la segunda posición en el ranking de la tabla 2b, pero no podría establecerse como un nodo objetivo de las propuestas de actuación puesto que existe por debajo de él un nivel causal más desagregado y concreto que constituye la «puerta de entrada» a su transformación (nodos: Falta asociacionismo [72], Falta comunicación entre Ayuntamiento y población [81], Falta de información del Ayuntamiento a la población [93] e Individualismo his- 
tórico [131]). Así, entre los nodos que muestra el indicador cercanía ejercida es necesario realizar una criba de carácter cualitativo. Es decir, en el proceso de análisis se debe identificar los nodos más influyentes y decidir cuáles tienen un nivel adecuado de concreción y operatividad para poder actuar directamente sobre ellos desde el ámbito local. En otros casos, como con el nodo Clima [32], simplemente las causas escapan al ámbito local por lo que el nivel de desarrollo al que se ha llegado en el mapa causal está justificado. No obstante, puede ocurrir que ciertos nodos sean definidos por la estructura relacional y señalados por el análisis estadístico como centrales, pero que no alcancen el nivel necesario de concreción y operatividad. En este caso habría que reanudar el mapa causal de este nodo hasta dar con el nivel de concreción requerido. Por ejemplo, sería necesario profundizar en las causas de Falta de asociacionismo [72] para poder emprender medidas de actuación que redunden en una mayor participación de la ciudadanía. De darse esta participación, se generaría una amplia onda expansiva (debido a la elevada cercanía ejercida de este nodo) en el turismo residencial en Dénia.

La escasa concreción y operatividad de algún nodo puede suscitar alguna duda sobre la validez de la información que arroja el mapa estructural de impactos. En este sentido, resulta evidente que el mapa causal puede ser desarrollado en mayor medida pudiendo generar un entramado relacional más amplio que quizás señale hacia otras causas de fondo con una mayor centralidad. Es decir, parece lógico esperar cambios en la centralidad de la red de impactos a partir de la incorporación de nuevos datos. Sin embargo, puede también ocurrir que la profundización en el mapa causal sólo evidencie las causas concretas de categorías más amplias pero sin implicar nuevas relaciones y cambios en la centralidad. En este segundo caso, resulta más eficaz concentrar los esfuerzos en el desarrollo de la línea causal de los nodos que adquieren relevancia en el contexto del caso de estudio y sus objetivos prioritarios. Sin embargo, ésta es una cuestión que en la mayoría de ocasiones no se descubre hasta que no se realiza este primer análisis.

En cualquier caso, los límites en el desarrollo de los mapas causales derivarán de 1) el tiempo y los recursos materiales y humanos que se pueda destinar a la elaboración de los mapas causales, y 2) el ámbito de actuación de los destinatarios de la investigación. En este caso, dado que este proyecto estaba dirigido a proporcionar información a los tomadores de decisiones del municipio de Dénia, los mapas causales se circunscribieron a la esfera local.

\section{CONCLUSIONES}

El uso de la herramienta informática de análisis de redes (Ucinet $\left.{ }^{\circledR}\right)$ permite realizar un análisis sofisticado del complejo entramado de impactos inducido por el turismo residencial. Este método identifica de forma sistemática los nodos con mayor potencia explicativa de la red. Los resultados obtenidos facilitan la ela- 
boración de itinerarios de actuación por parte de los tomadores de decisiones en el ejercicio del planeamiento local. Estos agentes pueden seleccionar los nodos más adecuados sobre los que intervenir a partir de los resultados del análisis estadístico de la red y de las circunstancias particulares de cada municipio.

Hasta la fecha, la mayoría de los diagnósticos realizados sobre municipios turístico-residenciales habían identificado la Escasa e inadecuada planificación urbanística como la principal causa de los problemas que aquejan a este tipo de municipios (MUNRES, 1995; Navalón, 1995; Vera, 1997; Mazón, 2006). No obstante, el uso de Ucinet ${ }^{\circledR}$ y NetDraw ${ }^{\circledR}$ para analizar de forma conjunta los mapas causales aporta unos resultados distintos en el caso del diagnóstico de los impactos inducidos por del turismo residencial en Dénia. Si se hubiera tenido en cuenta exclusivamente las influencias directas, habría aparecido de nuevo la Escasa e inadecuada planificación urbanística como el nodo con mayor capacidad explicativa de la red. Esto es así porque este nodo ejerce el mayor número de influencias directas (10) sobre otros nodos (Ver tabla 2a).

Sin embargo, cuando se observan los nodos más influyentes y explicativos de la red a partir del análisis de las influencias indirectas ejercidas, se comprueba que la Escasa e inadecuada planificación urbanística pierde peso explicativo. Aparecen, entonces, otros nodos más influyentes que hacen referencia al déficit democrático (Morlino, 2003) existente durante los procesos de toma de decisiones en el ejercicio de las potestades municipales en materia de urbanismo y planificación. En concreto, los nodos con una mayor capacidad explicativa del sistema relacional se encuentran alrededor (son causa) del nodo Falta de participación ciudadana (la segunda en valoración) y hacen referencia a la Falta de asociacionismo, a la Falta de comunicación entre Ayuntamiento y población, a la Falta de información del Ayuntamiento a la población y al Individualismo histórico. Así, la aplicación de este método revela que el nodo que, en un primer momento, podría considerarse como un nodo causal sobre el que dirigir las actuaciónes de forma prioritaria (p.e. Escasa e inadecuada planificación urbanística), resulta ser más bien un síntoma ocasionado por una serie de causas más basales.

Así, mientras esta propuesta comparte con otros métodos de diagnóstico las limitaciones de reducir la realidad a un número de nodos y de sus relaciones, este método aporta una serie de virtudes relacionadas con: 1) la mayor cantidad y calidad de la información de base mediante la incorporación de las partes interesadas, y 2) una herramienta sofisticada de gestión de la información que permite llevar a cabo análisis holísticos del segmento de la realidad seleccionado. De esta manera se proporciona a los tomadores de decisiones locales una herramienta para la planificación que desvela la intrincada red causa-efecto generada por el turismo residencial partiendo de una información altamente valiosa y rica en contendidos por su origen deliberativo y socialmente compartido. 


\section{GLOSARIO}

Impactos Impactos priorizados por los stakeholders de Dénia. Constituyen el punto de partida de los mapas causales.

Nodos Término para designar las causas generadoras de los impactos que aparecen en los mapas causales.

Cercanía Mide la distancia media de cada nodo con respecto al resto de no$d o s$ de la red. Son más centrales en la red los nodos con un mayor índice de cercanía debido a que tienen que dar un menor número de pasos para relacionarse con el mayor número de nodos de la red. En el programa Ucinet ${ }^{\circledR}$ aparece como Closeness.

Grado Mide el número de relaciones directas de cada nodo de la red. En el programa Ucinet ${ }^{\circledR}$ aparece como Degree.

\section{BIBLIOGRAFÍA}

ACKERMAN, F., y EDEN, C. (2001): «Contrasting single user and Networked Group decisión support systems for strategy making», Group Decisión and Negotiation,10, pp. 47-66.

Albino, V., KüHTZ, S. y ScozZI, B. (2002): Cognitive maps and the

sustainable development in the Mediterranean region, Proceedings of local resources and global trades. INCOSUSW Meeting, Rabat (Morocco). Disponible en http://ressources.ciheam.org/om/pdf/a57/04001986.pdf [consulta]

ALEDO, A. (2008): «De la tierra al suelo: la transformación del paisaje y el nuevo turismo residencial» Arbor, 184, 729, pp. 99-113.

ALEDO, A. y MAZÓN, T. (2005): «Impacts of residential tourism and the destination life cycle theory», en F. D. Pineda (ed.), Sustainable Tourism, Wessex, Witpress, pp. 2536.

Almenar, R. (2000): La Sostenibilidad del Desarrollo: el caso valenciano. Valencia. Universidad de Valencia - Fundación Bancaja.

AP, J. (1992): «Residents' Perceptions on Tourism Impacts», en Annals of Tourism Research, 19.

ARrochA, A. (2005): «La hacienda local en los destinos turísticos: el caso de Torrevieja», en T. Mazón y A. Aledo, Turismo residencial y cambio social. Alicante, CAM-UA.

BECKER, H. A., y VANCLAY, F. (2003): The international handbook of social impact assessment: Conceptual and methodological advances. Cheltenham: Edward Elgar Pub.

Benson, M. (2009): Lifestyle Migration. Farnham, Ashgate.

Bramwell B., Sharman A. (1999): «Collaboration in Local Tourism Policymaking» Annals of Tourism Research, 26, 2, pp. 392-415.

Brohman, J. (1996): Popular Development: Rethinking the theory and practice of development. Oxford. Blackwell. 
Burdge, R (2004): The Concepts, Process and Methods of Social Impact Assessment. Middelton (WI): Social Ecology Press.

CAÑADA, E. (2010). Turismo en Centroamérica,nuevo escenario de conflicto social. Managua, AlbaSud.

CASAdo DíAZ, M.A. (1999): «Socio-demographic impacts of residential tourism: a case study of Torrevieja». The International Journal of Tourism Research, 1, pp. 223-237.

CiUdAD, R., (1995): «Población y desarrollo urbano de la ciudad de Dénia». Espacio, Tiempo y Forma, 6. 8, pp. 35-99.

CoHEN, J. (1997): Deliberation and democratic legitimacy en Deliberative democracy: Essays on reason and politics J. Bohman, \& W. Rehg (Eds) Cambridge,MA, The MIT Press, pp. 67-91.

Cossette, P., AUdET, M. (1992): «Mapping of an idiosyncratic schema», Journal of Management Studies. 29, 3, pp. 325-347.

CopPOCK, J. (1977): Second homes: curse or blessing?. Oxford. Pergamon Press.

Da Silva, A. Ferreira, A. (2007): Dinâmica imobiliária e turismo: Novas relações, novos riscos. Cadernos Metrópole, 18, 109-133.

Díez Ripollés, J.L., Gómez-Cespedes, A., Prieto Del Pino, A. M., Stangeland, P y VERA JURADO, D., (2003): «Urbanismo, corrupción y delincuencia organizada: un proyecto en la Costa del Sol», en Boletín Criminológico, 66,

DíEz RIPOLlÉS, J.L., GóMEZ-CESPEDES, A., (2008): «La corrupción urbanística: Estrategias de análisis», en Revista Española de Investigación Criminológica, 6, 5.

ElSTER, J. (1998): La democracia deliberativa. Barcelona: Gedisa Ed.

Evans, J.R. (2005): Total quality management, organization, and strategy, 4⿳亠丷a edición, Mason, OH: Thomson South-Western.

EXCELTUR (Alianza para la excelencia turística) (2005): Impacto de los modelos de desarrollo turístico en el litoral mediterráneo y las islas, Madrid. Dirección URL: [Consulta: 22 de agosto de 2007].

Ferreira, A. CARdoso, A. (2007): Perdas e ganhos na produção imobiliária: Uma agenda pública para o futuro. Scripta Nova, 12, 245, p. 44.

Funtowicz, S. y RAVETZ, J., [1993] (2000): La ciencia posnormal. Icaria. Barcelona.

GARCIA-ANDREU, H. (2005): «Un acercamiento al concepto de turismo residencial», en MAZON, T. y Aledo, A., Turismo residencial y cambio social: nuevas perspectivas teóricas y empíricas. Alicante. Agua Clara, pp. 55-70.

-(2008): «SOCIEDAD, TURISMO Y MEDIO AMBIENTE: Una propuesta desde la Sociología para el diagnóstico y la reorientación de los municipios turístico-residenciales del litoral español». Tesis Doctoral. Dpto. de Sociología I. Universidad de Alicante. Defendida el 29 de febrero de 2008.

GARCIA-ANDREU, H. y RoDES, J. (2004): «Análisis y comparativa de cinco municipios turísticos de la Costa Blanca», en ALVAREZ SOUSA, A. (coord.), Turismo, ocio y deporte. A Coruña. Universidad A Coruña, pp. 377-392.

GARTNER, W. (1987) Environmental impacts of recreational home development. Annals of Tourism Research, 14, pp. 38-57,

GILMORE, M. J., y MuRPHY, J., (1991): «Understanding classroom environments: An organizational sensemaking approach», Education Administration Quarterly, 27, 3, 392-429.

Grenon, M. (1990): El plan azul : el futuro de la Cuenca Mediterránea, Ministerio de Obras Públicas y Transporte: Madrid,

GREENPEACE (2007): «Destrucción a toda costa: informe sobre la situación del litoral español», disponible en [consulta: 9-9-2007] 
GunN, C.A. (1994): Tourism Planning: Basics, Concepts, Cases. Taylor and Francis. Washington.

Hall, C. (2004): Tourism, Mobility and Second Homes: Between Elite Landscape and Common Ground (Aspects of Tourism). Clevedon. Channel View Publications.

HANNIGAN, J. (1995): Environmental sociology: a social constructionist perspective. Londres. Routledge.

Hemmati, M. (2002): Multi-stakeholder Processes for Governance and Sustainability. Londres, Earthscan.

JAMAL, T. y GETZ, D. (1995): «Collaboration Theory and Community Tourism Planning», en Annals of Tourism Research. 22, 1, pp. 186-204.

JiMÉNEZ SÁNCHEZ, S. (2008): «Boom urbanístico y corrupción política en España», en Mediterráneo Económico, N. ${ }^{\circ} 14$. disponible en.

Jurdao Arrones, F. (1992): Los mitos del turismo. Madrid. Endymion.

KeOGH, B. (1990): «Public Participation in Community Tourism Planning» Annals of Tourism Research, 17, 3, pp. 449-65.

KING, R. (2000): Sunset Lives: British Retirement Migration to the Mediterranean. Londres. Berg Publishers.

LANE, B. (1994): Sustainable Rural Tourism Strategies: A Tool for Development and Conservation en Rural Tourism and Sustainable Rural Development, B. Bramwell

and B. Lane, eds. Clevedon, Channel View, pp. 102-111.

LANKFORD, S.V. (1994): «Attitudes and Perceptions toward Tourism and Rural Regional Development». Journal of Travel Research, 32.

LonG, .H. (1993): Techniques for Socially Sustainable Tourism Development: Lessons from Mexico, en Tourism and Sustainable Development: Monitoring, Planning, Managing, Nelson, J.G. Butler, R.W. y Wall, G.(eds.) Department of Geography, University of Waterloo. Waterloo.

MCWATTERs, M. (2009): Residential Tourism: (De) constructing paradise. Bristo. Channel View Publications

Manin, B., Strein, E., Mansbridge. J. (1987): «On legitimacy and political deliberation». Political Theory, 15,3 , pp. 338-368.

MANTECÓN, A. (2005): «La construcción social de la experiencia turística» en Turismo residencial y cambio social: nuevas perspectivas teóricas. Alicante, CAM, Obras Social y Universidad de Alicante, pp. 391-410.

MATHIESON, A y WALL, G. (1990): Turismo: Repercusiones económicas, físicas y sociales. México. Trillas.

Mazón, T. (2006): «Inquiring into Residential Tourism: The Costa Blanca Case». Tourism and Hospitality Planning \& Development, 3, 2, pp. 89-97.

MAzón, T. y Huete, R. (2005): «Turismo residencial en el litoral alicantino: los casos de Dénia, Altea, Benidorm, Santa Pola y Torrevieja», en MAZON, T. y ALEDO, A, Turismo residencial y cambio social. Nuevas perspectivas teóricas y empíricas. Alicante. Aguaclara.

Mazón, T., Aledo, A., Arrocha, A. y Martínez, E. (1996): El turismo inmobiliario en la provincia de Alicante: análisis y propuestas. Alicante. Patronato Provincial de Turismo Costa Blanca, Diputación de Alicante.

MERINERO, R., y PULIDO, J.I. (2009): «Desarrollo turístico y dinámica relacional: metodología de análisis para la gestión activa de destinos turísticos», en Cuadernos de turismo, N. ${ }^{\circ} 23$, pp. 173-194. 
Miles, M. B. y Huberman, A. M. (1994): Qualitative data analysis: an expanded sourcebook. Londres. Sage.

Mitchelll, R, Agle, B, \& Wood, D. (1997): Toward a theory of stakeholder identification and salience: Defining the principle of who and what really counts. The Academy of Management Review, 22, 4, p. 853.

MonReAl, J. (dir.) (2001): Un nuevo mercado turístico: jubilados europeos en la Región de Murcia. Universidad de Murcia. Murcia.

MoretTI, A. (1997): «Le mappe cognitive», in Costa G., Nacamulli R.C.D. (eds.) Manuale di organizzazione aziendale, Vol.2, Torino, Utet.

Morlino, L (2003): Democrazie e democratizzazioni, Bologna, Il Mulino, pp 184-206.

MunRes (1995): Programa de revitalización de municipios con turismo residencial. Alicante. Diputación Provincial de Alicante.

MurPhy, P.E. (1985): «Tourism: a Community Approach». Routledge. Londres.

NASH, R., (2006): Casual Network Methodology. Tourism Research Applications, en Annals of Tourism Research, 33, 4, pp. 918-938.

NAVAlon, M. (1995): El planeamiento urbano y el turismo residencial en los municipios litorales alicantinos. Alicante. Ed I.E. Juan Gil-Albert.

NinO, C. (1996): The constitution of deliberative democracy. New Haven: Yale University Press.

Nogués-PedRegal, A. (2010): Poder político local y urbanismo en entornos turísticos. La mediación del espacio turístico en la producción de significados disponible en http://www.ugr.es/ pwlac/Welcome2008.html [consulta]

OlcinA, J. y Rico, A. (2005): «Un crecimiento territorial con pies de barro», en J.

Romero y M. Alberola (coords.), Los límites del territorio. El País Valenciano en la encrucijada. Valencia. Universidad de Valencia.

Ortiz, G., Garcia-Andreu, H., Juan, Ma . P., Aledo, A. (2007): «Epistemological and ethical dilemmas of public participation on residential tourism planning», en Pasos. Revista de turismo y Patrimonio Cultural, 5, 3, pp. 323-329.

OSEa (Observatorio de la Sostenibilidad en España) (2006): Sostenibilidad en España 2006, Madrid, Mundi-Prensa Libros S.A.

OSEb (Observatorio de la Sostenibilidad en España) (2006): Cambios de ocupación del suelo en España, Madrid, Mundi-Prensa Libros S.A.

RODRÍGUEZ, J.A. (1995): Análisis estructural y de redes. CIS. Madrid.

- (2000) «El círculo del poder: La estructura social del poder económico en la España de los noventa», en Sistema, 158.

Rodríguez Diaz, J., García Andreu, H., José Vílchez, F., Moltó Seguí, E., Sempere, F. (2004): «La red terrorista del 11-M», en REIS, 107, pp. 155-179.

Rodríguez, V., Fernández-Mayoralas, G. y RoJo, F. (1998): «European retired in the Costa del Sol: A cross-Nacional Comparison», en International Journal of Population Geography, No 4.

Ros, J. (2003): «Aproximación al turismo residencial español», en Estudios Turísticos. $155, \mathrm{pp} 71-86$.

Scott, N. Baggio, R. Cooper, C. (2008): Network Analysis And Tourism. From Theory To Practice. Clevedon, Channel View.

STOLP, A. (2003): «Citizen values assessment» en The international handbook of social impact assessment. Cheltenham, Edward Elgar. pp. 231-257.

TORRES, E. (2003): «El turismo residenciado y sus efectos en los destinos turísticos». Estudios Turísticos, 155-156:, p. 45. 
Tosun, C. y Timothy, D. J. (2003): «Arguments for Community participation in the tourism development process», en The Journal Of Tourism Studies, 14.

TRANSPARENCY INTERNATIONAL ESPAÑA (2006): Informe Global de la Corrupción 2006. Disponible en http://www.transparencia.org.es/INFORME\%20GLOBAL\%202006 /Informe\%20TI-España\%202006.pdf

Tufte, E. (2001): The Visual Display of Quarantine Data. Providence, RI, Graphics Press.

VANClay, F. 2000. «Social Impact Assessment». Prepared for Thematic Review V.2: Environmental and Social Assessment for large dams. Center for Rural Social Research, Charles Stuart University, Australia. $<$ http://www.agsci.utas.edu.au/WCDreportSIA\&dams.pdf $>$. [9 de febrero de 2010]

VERA, F. (1992): «Turismo y crisis agraria en el litoral alicantino», en JURADO, F., Los mitos del turismo. Madrid. Endimión, Col. Turismo y Sociedad.

-(coord.) (1997): «La dimensión territorial y ambiental del turismo», Análisis territorial del turismo: una nueva geografía del turismo, Barcelona. Editorial Ariel.

VERA, F. y MONFORTE, V. (1994): «Agotamiento de modelos turísticos clásicos. Una estrategia territorial para la cualificación: la experiencia de la Comunidad Valenciana», en Revista Estudios Turísticos, 123.

Viruela, R. y Domingo, C. (2001): «Población extranjera en el País Valenciano». Arxius, 5, pp.147-182.

WARnES, A. (2001). «The International Dispersal of Pensioners from Affluent Countries», International Journal of Population Geography. 7, 5, pp. 373-388.

Weber, P. S., y MichaEL, R. M., (2001): «Cause maps, sensemaking and planning organizational change», Journal of Applied Behavioral Science, 37, 2, pp. 227-251.

WeIcK, K. E., (1979): The Social Psycology of Organizing. New York: McGraw-Hill.

Williams, A. (1997): A Place in the Sun: International Retirement Migration from Northern to Southern Europe. European Urban And Regional Studies, 4, 2. pp. 115-134.

WINN, M. I. (2001): Building stakeholder theory with a decision modeling methodology Business \& Society, 40, 2, pp. 133-166.

Woodside, A. (2009). Applying Systems Thinking to Sustainable Golf Tourism. Journal of Travel Research. 48, 2 pp. 205-214.

\section{RESUMEN}

Este artículo presenta la novedad metodológica de aplicar los programas informáticos de análisis de redes sociales (Ucinet ${ }^{\circledR}$ y Netdraw ${ }^{\circledR}$ ) al análisis de mapas causales con la finalidad de mejorar los procesos de diagnóstico del turismo residencial a escala local. En concreto, esta propuesta metodológica se articula en los siguientes pasos: primero, la identificación de los impactos a escala local y la elaboración de sus mapas causales con la participación de actores locales relevantes; segundo, el análisis de redes de los mapas causales. Mediante este análisis es posible identificar las variables de mayor repercusión sobre las estructuras ambiental, económica y social de los municipios turísticos. Esta propuesta metodológica consigue sistematizar un gran volumen de información que ayuda al planificador local a discriminar las variables más influyentes con las que 
elaborar itinerarios de actuación más rentables en términos de inversión de recursos. El caso de estudio es el municipio de Dénia, situado en el norte de la provincia de Alicante. Los resultados muestran que las causas profundas de los impactos negativos inducidos por el turismo residencial están conectados con el déficit democrático en el proceso de gestión municipal del desarrollo turístico-residencial.

\title{
PALABRAS CLAVE
}

Metodología de Redes Causales, Turismo, Impactos, Diagnóstico.

\begin{abstract}
This paper presents the methodological innovation of applying the social network analysis software (Ucinet ${ }^{\circledR}$ and Netdraw ${ }^{\circledR}$ ) to the Causal Networks Methodology (CNM) with the aim of improving the processes of diagnosis on the tourism activity functioning at a local scale. Specifically, this methodological proposal is structured in the following steps: first, the identification of the impacts of the residential tourism activity and the creation of their causal chains with the participation of local stakeholders; second, the analysis of the network of causal chains. This analysis allows the identification of the variables with the highest level of repercussion on the environmental, economic and social structures at the tourist municipalities. This methodological proposal manages to systematize a high volume of information and helps the local planner to discriminate the most influencing variables for their use in the design of more efficient lines of action in terms of resource investment. The selected case of study is the municipality of Denia, on the North of the province of Alicante. The results show that the deep causes of the negative impacts induced by the residential tourism are connected to a democratic deficit in the local management activity regarding residential tourism development.
\end{abstract}

\section{KEY WORDS}

Causal Networks Methodology, Tourism, Impacts, Diagnosis. 